\title{
Analysis of the bending of a neo-Hookean electro-elastic shell of arbitrary thickness under an externally-applied hydrostatic pressure
}

\author{
Omid Teymooria (i), Ali Hatami* ${ }^{a *}$
}

aDepartment of Mathematics, Statistics and Computer Sciences, University of Sistan and Baluchestan, Zahedan, Iran. E-mails: ahatami@math.usb.ac.ir, omid.teymoori@pgs.usb.ac.ir

*Corresponding author

https://doi.org/10.1590/1679-78256692

\begin{abstract}
The present study analyzes the bending of a simple electro-elastic cylindrical shell by the compound matrix method. The cross-section of the circular cylindrical shell is a non-circular curved shape, with $\mu_{1}$ a function of $A / B$ and the mode number, where $A$ and $B$ are the pre-deformation inner and outer radii of the cylindrical shell, and $\mu_{1}$ is the ratio of the deformed inner radius to $A$. In the first step, a numerical model of the problem is developed to obtain specific differential equations. The modeling yields a system of two Ordinary Differential Equations with three boundary conditions of the same type. Next, it is shown that the dependence of $\mu_{1}$ to $A / B$ has a boundary layer structure. Simple numerical observations were made for bifurcation conditions. The analysis is, in fact, based on the variations of the inner and outer radii $A$ and $B$, assuming $a=$ $\mu_{1} A$ and $b=\mu_{2} B$, and based on the bifurcation of $\mu_{1}$ and $\mu_{2}$ ratios with respect to radius. For this purpose, the compound matrix method is used to show the validity of the arguments.
\end{abstract}

\section{Keywords}

Nonlinear Electro-elasticity, Compound Matrix Method, Bending Bifurcation, Finite Deformations, Electric Field

\section{Graphical abstract}
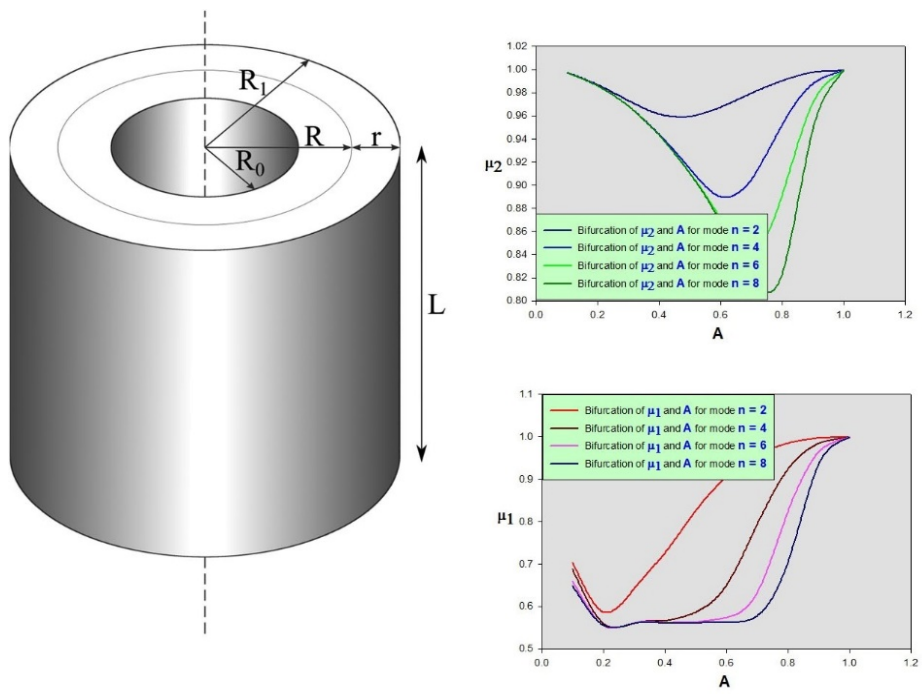


\section{Introduction}

The study of nonlinear electro-elasticity is a major branch of physics and mathematics in solid mechanics and statics. In the age of nanotechnology and bioengineering, much research is centered on multipurpose applications in solid and fluid mechanics. One topic of interest in mechanical engineering (solids and fluids) is the theory of hyperelasticity, Dorfmann and Ogden (2005). The study of elastic and electro-elastic materials is within the scope of nonlinear elasticity and electroelasticity. The materials have several applications, including in motor technologies, sensors, imaging systems, smart adaptive structures, and artificial muscle. Further, these materials have been incorporated into complex and heterogeneous systems such as granular media and composites. It must be noted that the term Maxwell stress originates in the electrostatic field, with the elastic and electrical states of the object intrinsically coupled. The elastic and electrical behavior of the object is significant in establishing general nonlinear constitutive rules. Accordingly, most authors present these constitutive rules and relations in the specific form of a mathematical and physical model, Fiebig (2005) and Toupin (1956(.Toupin (1956) set the groundwork for a general theory on electro-elastic materials, Fiebig (2005) and Toupin (1956). By including the effects of heat, Tiersten (1971) further developed the problem. Later, Zharii (1990) formulated the general expansion of the Eigen-function in non-stationary dynamic problems based on nonlinear and linear electro-elasticity theories. The extensive use of piezo-electric converters requires the behavior of piezo-ceramic structural members to be studied, Dieulesiant , Royer (1981) and Shul'ga (2005). In this regard, comprehensive studies were carried out on harmonic electro-elastic vibrations of piezo-ceramic (solids Dieulesiant, Royer (1981) and Shul'ga (2005)).

Eva (2006) tested different methods for establishing numerical algorithms for electro-elasticity, eventually solving the stationary vibration problem for a radially-polarized hollow piezo-electric cylinder under different electrical boundary conditions and mechanical pressure. Later, Eva and Shul'ga (2008) relied on the Hamilton - Starogradski principle to address a particular case of initial-boundary-value problems in the theory of electro-elasticity. They considered the problem of free vibrations of a piezo-ceramic layer to characterize non-stationary problems in the theory of electroelasticity. Electro-sensitive elastomers often referred to as electro rheological elastomers are materials that react to an electric field by changing their mechanical behavior considerably. Thanks to their potentials, these materials have recently received much attention, Dorfmann and Ogden (2005). The Eigen-function Expansion Method (EEM) can be used to obtain some general properties of non-stationary wave fields in quasi-elastic wave. Recent successes in new dielectric elastomer material technology have led to the rapid rise of devices relying on electro-sensitive material, including actuators, sensors, energy conversion equipment, and making artificial muscle prototypes, Melnikov and Ogden (2016). Elastic electro-sensitive polymers are materials, whose mechanical properties can be rapidly tuned using an electric field. Showing remarkable potential for making chip connections and replacing lamps in mechanical devices such as actuators, these materials have evoked much scientific attention among industries, companies, and individuals thanks to scientific advances in bioengineered tissues and artificial muscle. Electro-active Polymers (EAPs) are materials that react to electrostatic actuation by deformation. Offering light weight and flexibility, these accessible materials have several applications, including artificial muscle (Bar-Cohen (2001)), energy generation equipment (Mckay et al. (2010)), micro pumps (Rudykh et al. (2012)), and tunable wave guides (Shumel et al. (2012)), among others. Dorfmann and Ogden (2010) proposed a formulation, showing the dependence of the surface wave velocity on the electric field in an electro-elastic half-space (voltage-controlled). Shumel et al. carried out a study on the dielectric layer under plane strain, showing the effects of the bias electro-static field and pre-stretch on the wave velocity under load control, (Shumel et al. (2012)). In a more recent study, Wang et al. (2017) investigated vibrations in compact multi-layer plates, discussing interesting phenomena, such as frequency veering in plates under pressure.

Several works have been published in recent years, including those by Dorfmann and Ogden (2017), on the study and introduction of the theory of nonlinear electro-elasticity and electro-elastic materials. Dorfmann and Ogden (2017) took a theoretical approach to the analysis of boundary value problems, presenting applications for certain electromechanical reactions. Dorfmann and Ogden (2005) provided the theoretical grounds for characterizing the nonlinear features of electroelastic materials, applying the theory in some boundary value problems for initial models comprising simple electro-elastic materials, while showing the effects of the impact of the electric field on mechanical reactions of materials, Dorfmann and Ogden (2005). They investigated the effect of a radial electric field on the axial shear of a thick-walled circular cylindrical tube, describing nonlinear electromechanical reactions in shape-shifting materials and summarizing the required relations from nonlinear continuum mechanics, Melnikov (2016).

Bifurcation of an internally-pressurized, thick-walled, circular, elastic shell was addressed by Liu (2014) under perfectly elastic conditions. Recently, more results have been collected on different elastic models, including details regarding external pressure. Melnikov and Ogden (2016) later investigated the bifurcation of deformations in a pressurized, thick-walled, circular, cylindrical tube made of a soft dielectric material that was closed on both ends and had compliant electrodes on its curved boundaries, analyzing the configuration based on the theory of electro-elasticity, 
Melnikov et al. (2020). Later, Melnikov (2016) wrote a thesis on the finite deformations of a pressurized, circular, cylindrical electro-elastic tube under an electric field and with compliant electrodes on boundaries. He also explained the dependence of pressure and reduced axial load in the deformation, showing the voltage between electrodes or the uniform surface charge distribution, Haughton and Ogden (1978).

Further expanding their works, Melnikov and Ogden (2018) studied prismatic bifurcations of a deformed, thickwalled circular, cylindrical tube of electro-elastic material in a radial electric field, finding the number of possible modes for a stretched neo-Hookean electro-elastic material and the axisymmetric bifurcation of neo-Hookean and Mooney Rivlin electro-elastic materials Haughton and Ogden (1978). Finally, together, Melnikov et al. (2020) examined a pressurized spherical electro-elastic shell with compliant electrodes on inner and outer surfaces. Drawing on the theory of small incremental electro-elastic deformations, they obtained a new configuration for the spherical shell. In the study of electro-active materials, Lie (2014) presented an energy formulation pf continuum electro-elasticity based on the freeenergy principle. He proposed a general form of total free energy as a three-dimensional system of equations, implicitly deriving linear and nonlinear boundary value problems for electro-elastic and electro-magneto-elastic materials based on the free-energy principle and from Euler-Lagrange equations. Cohen et al. (2016) undertook a multi-scale analysis of electromechanical coupling in elastic dielectrics. Their analysis started from the discrete monomer level and extended to the polymer chain at the macroscopic level. Accordingly, three models were developed simulating the local relations between molecular dipoles and their corresponding electric fields, introducing the electro-elasticity of polymer networks as a new concept (Sanjaranipour et al. (2013)). Expanding on their previous works, Su et al. (2019) addressed deformations caused by bending a dielectric-elastic bilayer reacting to voltage variations. Relying on the theory of nonlinear electro-elasticity and linearized incremental field theory, they carried out numerical and linear analyses. Broderick et al. (2020) studied the Lamb wave propagation in soft dielectric plates under electrical and mechanical loading. They also discussed explicit expressions for the dispersion equations for neo-Hookean and Gent dielectrics, explaining field effects on the plate thickness and wavelength Broderick et al. (2020).

The present study is structured as follows:

Section 2 is dedicated to the concepts and structural equations of nonlinear electro-elasticity. In Subsection 2.2, boundary conditions and governing equations are discussed. Under Section 2.2, the Lagrangian form of electric field components is presented, Subsection 2.3 defines and formulates structural equations. 2.4 investigates finite deformations in a hollow, cylindrical tube of neo-Hookean electro-elastic material.

Section 3 goes into the bifurcation analysis of the hollow cylindrical shell made of neo-Hookean electro-elastic material. Further, spectral bifurcations and boundary conditions are discussed in Sections 3.1 and 3.2, and the problem is non dimensionalized in Section 3.3.

In the end, Section 4 goes through the analysis of numerical results using the compound method, presenting the data in tables and figures.

\section{Nonlinear Electro-elasticity Equations}

Let us assume an electro-elastic, deformable solid with a reference configuration of $\mathcal{B}_{r}$ and boundary conditions of $\partial \mathcal{B}_{r}$ in the absence of any body and hydrostatic forces or electric fields. By applying specific body and hydrostatic forces and subjecting the object to an electric field, the new configuration $\mathcal{B}$ with boundary condition $\partial \mathcal{B}$ is formed, which is referred to as the current configuration. By assuming $X$ and $x$ denote positive vectors in the reference $\mathcal{B}_{r}$ and current $\partial \mathcal{B}$ configurations, the location of the particle and the deformed object in the current configuration can be expressed as $x=\chi(X)$. Now, the deformation gradient tensor $F$ is defined as follows:

$\mathrm{F}=\operatorname{Grad}(\chi)$,

where Grad is the gradient operator defined based on $\chi$. Further, in the absence of body forces, the relation below holds:

$\mathrm{J}=\operatorname{det} \mathrm{F}=1$,

This relation, known as the incompressibility condition, must hold for any $X$. Depending on the deformation gradient, left and right Cauchy-Green deformation tensors, defined in the following, must be used:

$C=F^{T} F, B=F F^{T}$. 


\subsection{Governing Equations and Boundary Conditions}

The Maxwell equation for dielectrics is considered in the following forms in statics:

Curl $\mathrm{E}=0, \operatorname{div} \mathrm{D}=0$,

where $E$ is the electric field and $D$ is the electric displacement vector in the current configuration $\mathcal{B}$. Further, Curl and div operators are defined based on $x$. The corresponding fields outside of the elastic object are represented by $E^{*}$ and $D^{*}$ between which the following standard relation holds for non-polarizable materials:

$D^{*}=\varepsilon_{0} E^{*}$,

where $\mathcal{E}_{0}$ is the vacuum permittivity. These parameters must satisfy (2.4), too. Then, according to (2.4), standard boundary conditions are as follows:

$\left(E^{*}-E\right) \times n=0,\left(D^{*}-D\right) \cdot n=\sigma_{F}$ on $\partial \mathcal{B}$,

where $n$ is a unit normal vector on the boundary of $\partial \mathcal{B}$ and $\sigma_{F}$ is the free surface charge on the said boundary per unit area. In the absence of body and mechanical forces, the mechanical equilibrium equation is simplified by joining electric field forces:

$\operatorname{div} \sigma=0$,

where $\sigma$ denotes the overall Cauchy stress tensor and depends on the deformation and electric field on the object. Let us not that $\sigma$ is symmetric. Moreover, the boundary conditions corresponding to (2.7) are as follows:

$\sigma \mathrm{n}=t_{a}+t_{m}^{*}$ on $\partial \mathcal{B}_{t}$

where $\partial \mathcal{B}_{t}$ is the stretched portion of the boundary, $t_{a}$ is the mechanical component of tension, and $t_{m}^{*}=\sigma_{m}^{*} n$ is the overstretch based on the Maxwell stress $\sigma_{m}^{*}$, which is calculated from the field outside object $\mathcal{B}$. Maxwell stress is calculated as follows:

$\sigma_{m}^{*}=\varepsilon_{0} E^{*} \otimes E^{*}-1 / 2 \varepsilon_{0}\left(E^{*} \cdot E^{*}\right) I$,

where $I$ represents the identity tensor.

\subsection{Lagrangian Form of Electric Fields}

The Lagrangian form of electric fields is defined as follows:

$E_{L}=F^{T} E, D_{L}=J F^{-1} D$

Note that $J=\operatorname{det} F$, and these relations must comply with the reference configuration.

$\operatorname{Curl} E_{L}=0, \operatorname{Div} D_{L}=0$.

Here, Curl and div operators are defined based on X. Further, by obtaining the Lagrangian forms of equilibrium equations (2.7), the nominal stress tensor is defined as follows.

$T=J F^{-1} \sigma$.

Further, according to the equation, the Lagrangian form of the electromechanical equilibrium equation (2.7) is as follows:

$\operatorname{Div} \mathrm{T}=0$, 
Further, the boundary conditions corresponding to (2.13) can be obtained from the relation below:

$\sigma \mathrm{nds}=T^{T} \mathrm{~N} \mathrm{dS}$

This relation was established using Nanson's formula $\mathrm{n} d \mathrm{~s}=\mathrm{J} F^{-T} N \mathrm{dS}$ for infinitesimal regions ds and dS in reference and current configurations, with $\mathrm{n}$ and $N$ representing unit normal vectors corresponding to the regions. Accordingly, (2.8) can be rewritten as follows:

$T^{T} \mathrm{~N}=t_{A}+t_{M}^{*}$ on $\partial \mathcal{B}_{r t}$,

where $\partial \mathcal{B}_{r t}$ is a pre-image of $\partial \mathcal{B}_{t}$ and $t_{A}$ and $t_{M}^{*}=T_{M}^{* T} \mathrm{~N}$ are mechanical and Maxwell stretch per unit reference area. Using Nanson's formula and (2.10), the boundary conditions of (2.6) are converted into the following Lagrangian form:

$\left(F^{T} E^{*}-E_{L}\right) \times N=0,\left(J F^{-1} D^{*}-D_{L}\right) . \mathrm{N}=\sigma_{F}$ on $\partial \mathcal{B}_{r}$

where $N$ denotes the normal unit on the boundary of $\partial \mathcal{B}_{r}$ and $\sigma_{F}$ are the free-surface charge density per unit area of $\partial \mathcal{B}_{r}$.

\subsection{Structural Equations}

Throughout this paper, $D_{L}$ is assumed as an independent electric variable. In this case, the overall stress tensor and electric field for incompressible electro-elastic materials are as follows:

$T=\partial W / \partial F-p F^{-1}, E_{L}=\partial W / \partial D_{L}$

where $W\left(F, D_{L}\right)$ is the total strain energy density function and is defined based on the type of elastic material. Further, $p$ is an arbitrary Lagrangian coefficient. For an incompressible, isotropic, electro-elastic material the strain energy function $W$ of which depends on $F$ and $D_{L}$, principal invariants are calculated based on the right Cauchy-Green deformation tensor $C$ and the electric displacement vector $D_{L}$ as follows:

$I_{1}=\operatorname{tr} C, I_{2}=1 / 2\left[(\operatorname{tr} C)^{2}-\operatorname{tr} C^{2}\right], I_{3}=\operatorname{det} C=1$,

$I_{4}=D_{L} \cdot D_{L}, I_{5}=D_{L} \cdot\left(C D_{L}\right), I_{6}=D_{L} \cdot\left(C^{2} D_{L}\right)$,

based on the definition of $W$, we aim to express it using principal invariants. Therefore, the stress $D_{L} \cdot\left(C D_{L}\right)$ and the electric field $E$ are defined:

$\sigma=2 W_{1} B+2 W_{2}\left(I_{1} B-B^{2}\right)-p I+2 W_{5} D \otimes D+2 W_{6}(D \otimes B D+B D \otimes D)$,

$\mathrm{E}=2\left(W_{4} B^{-1}+W_{5} \boldsymbol{I}+W_{6} B\right) D$,

where $W_{i}=\partial W / \partial I_{i}$ is calculated for $i=1,2,4,5$ and 6 , and $\mathcal{B}$ denotes the left Cauchy-Green tensor. The total nominal stress tensor $T$ is defined as follows:

$T=\boldsymbol{B} F+\boldsymbol{A} D_{L}+p F^{-1} F F^{-1}-p^{*} F^{-1}, E_{L}=\boldsymbol{A}^{T} F+\boldsymbol{C} D_{L}$,

where $\boldsymbol{B}, \boldsymbol{A}$, and $\boldsymbol{C}$ are tensors of the fourth, third, and second order, which are referred to as electro-elastic moduli based on $W$ and defined as follows:

$\boldsymbol{B}_{i j k l}=\partial^{2} W / \partial F_{j i} \partial F_{l k}, \boldsymbol{A}_{i j \mid k}=\partial^{2} W / \partial F_{j i} \partial D_{L_{k}}, \boldsymbol{C}_{i j}=\partial^{2} W / \partial D_{L_{i}} \partial D_{L_{j}} \cdot$ 
Since the tensor $T$ is symmetric, then $\boldsymbol{B}_{i j k l}=\boldsymbol{B}_{k l i j}, \boldsymbol{C}_{i j}=\boldsymbol{C}_{j i}$ and $\boldsymbol{A}_{i j \mid k}=\boldsymbol{A}_{j i \mid k}$. Therefore, (2.22), can be rewritten using these elements.

$$
T_{i j}=\boldsymbol{B}_{i j k l} F_{l k}+\boldsymbol{A}_{i j \mid k} D_{L_{k}}+p{F_{i j}}^{-1} F_{j k}{F_{k l}}^{-1}-p^{*}{F_{i j}}^{-1}
$$

$E_{L}=\boldsymbol{A}_{k j \mid i} F_{j k}+C_{i j} D_{L_{j}}$

\subsection{Finite Deformations of an Electro-elastic Tube}

Consider an incompressible, isotropic, homogeneous, and electro-elastic shell. Let us assume the electro-elastic shell is defined as follows in the reference configuration:

$A \leq R \leq B, 0 \leq \Theta \leq \pi, 0 \leq Z \leq L$,

where $A$ and $B$ are the inner and outer radii of the hollow electro-elastic cylinder and $L$ denotes its height. Applying an external hydrostatic pressure on the hollow cylinder deforms it, and the current shell configuration can be defined as follows:

$a \leq r \leq b, 0 \leq \theta \leq \pi, 0 \leq z \leq l$,

where $a$ and $b$ are the inner and outer radii subsequent to deformation (Dorfmann and Ogden (2005)). Note that the cylinder height remained unaffected. Therefore, a plane-strain deformation has taken place. The plane-strain deformation is defined as follows:

$r=r(R), \theta=\Theta, z=Z$,

In this case, the following relation holds between the inner and outer radii, before and after deformation:

$a=\mu_{1} A, b=\mu_{2} B$

where $\mu_{1}$ and $\mu_{2}$ are constants that satisfy the applied pressure problem, and $0 \leq \mu_{1}, \mu_{2} \leq 1$.

Moreover, the incompressibility condition is suggestive of the absence of body forces and charges. Accordingly, $\mu_{1}$ and $\mu_{2}$ are related as follows:

$\mu_{2}^{2}=1-\left(1-\mu_{1}^{2}\right)(A / B)^{2}$

Let $D_{r}(r)$ be the only element representing changes in the electric field. Here, $\operatorname{Div}(D)=0$, based on the electric displacement, holds and can lead to the following:

$\frac{d\left(r D_{r}\right)}{d r}=0$,

where $r D_{r}(r)$ is a constant. Therefore, for the boundary conditions of $r=a$ and $r=b$, we have:

$r D_{r}(r)=a D_{r}(a)=b D_{r}(b)$

According to Gauss's theorem, there is no field, then $D^{*}=0$. Now, the boundary condition of (2.6) on $=a$ and $r=b$ shows:

$D_{r}(a)=\sigma_{f a}, D_{r}(b)=\sigma_{f b}$,

where $\sigma_{f a}$ and $\sigma_{f b}$ denote the free-surface charge densities on the two boundaries. 


\section{Bifurcation Analysis of a Hollow Electro-elastic Cylinder}

This section investigates principal stretches in the deformation gradient tensor and bifurcation in the bending of a hollow cylindrical shell made of incompressible neo-Hookean material.

Let us assume that the displacement vector $x=\boldsymbol{u}$ can be written as follows:

$\boldsymbol{u}=v e_{1}+w e_{2}+u e_{3}$

Further, based basic vectors $e_{1}, e_{2}$ and $e_{3}$ on the component matrix $\boldsymbol{L}=\operatorname{grad}(\boldsymbol{u})$ is:

$\left[L_{i j}\right]=\left[\begin{array}{ccc}\frac{u+v_{\theta}}{r} & v_{z} & v_{r} \\ \frac{w_{\theta}}{r} & w_{z} & w_{r} \\ \frac{u_{\theta}-v}{r} & u_{z} & u_{r}\end{array}\right]$

The following can be derived from the incompressibility condition $\operatorname{tr}(\boldsymbol{L})=0$ :

$\frac{u+v_{\theta}}{r}+w_{z}+u_{r}=0$

\subsection{Spectral Bifurcations}

Assuming that $u, v$ and $w$ are independent of $z$, matrix $\boldsymbol{L}$ can be written for spectral bifurcations:

$\left[L_{i j}\right]=\left[\begin{array}{ccc}\frac{u+v_{\theta}}{r} & 0 & v_{r} \\ 0 & 0 & 0 \\ \frac{u_{\theta}-v}{r} & 0 & u_{r}\end{array}\right]$

Therefore, the incompressibility condition is reduced to:

$u+v_{\theta}+r u_{r}=0$

Given that $w=0$, the governing equilibrium equations are as follows in the electric field:

$T_{11,1}+T_{31,3}+\frac{1}{r}\left(T_{31}+T_{13}\right)=0$,

$T_{13,1}+T_{33,3}+\frac{1}{r}\left(T_{33}-T_{11}\right)=0$,

$\frac{\partial\left(r D_{L 3}\right)}{\partial r}+\frac{\partial\left(D_{L 1}\right)}{\partial \theta}=0$,

$\frac{\partial\left(r E_{L 1}\right)}{\partial r}-\frac{\partial\left(E_{L 3}\right)}{\partial \theta}=0$,

then, Cauchy stress components are calculated from the four equations above for the cylindrical configuration:

$T_{11}=\boldsymbol{B}_{1111} L_{11}+\boldsymbol{B}_{1133} L_{33}+p L_{11}-p^{*}+\boldsymbol{A}_{11 \mid 3} D_{L_{3}}$,

$T_{13}=\boldsymbol{B}_{1313} L_{31}+\boldsymbol{B}_{1331} L_{13}+p L_{13}+\boldsymbol{A}_{13 \mid 1} D_{L_{1}}$

$T_{31}=\boldsymbol{B}_{3131} L_{13}+\boldsymbol{B}_{3113} L_{31}+p L_{31}$,

$T_{33}=\boldsymbol{B}_{3311} L_{11}+\boldsymbol{B}_{3333} L_{33}+p L_{33}-p^{*}+\boldsymbol{A}_{33 \mid 3} D_{L_{3}}$, 
$E_{L_{1}}=A_{13 \mid 1} L_{31}+C_{11} D_{L_{1}}$

$E_{L_{3}}=\boldsymbol{A}_{11 \mid 3} L_{11}+\boldsymbol{A}_{33 \mid 3} L_{33}+\boldsymbol{C}_{33} D_{L_{3}}$.

The following relations are obtained by substituting these in (3.6) and (3.7), and using the incompressibility condition (3.5):

$r^{2} p_{r}^{*}=-\boldsymbol{B}_{1111} u+r\left(\boldsymbol{B}_{3333}+r \boldsymbol{B}_{3333}^{\prime}+r p^{\prime}\right) u_{r}+r^{2} \boldsymbol{B}_{3333} u_{r r}-2 \boldsymbol{B}_{1111} v_{\theta}+\boldsymbol{B}_{1111} u_{\theta \theta}+r\left(\boldsymbol{A}_{33 \mid 3}+\right.$

$\left.r \boldsymbol{A}_{33 \mid 3}^{\prime} D_{L_{3}}\right)+\boldsymbol{A}_{33 \mid 3} D_{L_{2}, r}+\boldsymbol{A}_{33 \mid 3} D_{L_{1}, \theta}$, (3.16)

$r p_{\theta}^{*}=r\left(\boldsymbol{B}_{3131}+r \boldsymbol{B}^{\prime}{ }_{31331}\right) v_{r}-\left(\boldsymbol{B}_{1111}+r p^{\prime}\right) v+r^{2} \boldsymbol{B}_{3131} v_{r r}+\boldsymbol{B}_{1111} v_{\theta \theta}+\left(2 \boldsymbol{B}_{1111}+r p^{\prime}\right) u_{\theta}+\frac{1}{2} \boldsymbol{A}_{33 \mid 3} D_{L_{1}}$,

Further, substituting (3.14) and (3.15) in (3.9) yields:

$-\boldsymbol{A}_{33 \mid 3}^{\prime} v+2 r D_{L_{1}} \boldsymbol{C}_{11}^{\prime}-\boldsymbol{A}_{33 \mid 3} v_{r}+2 \boldsymbol{C}_{11}\left(D_{L_{1}}+D_{L_{1}, r}+D_{L_{3}, \theta}\right)+\boldsymbol{A}_{33 \mid 3}^{\prime} u_{\theta}-+\boldsymbol{A}_{33 \mid 3} u_{r \theta}=0$.

\subsection{Boundary Conditions}

This section aims to find boundary conditions for the bending problem. Since $E^{*}=0$, according to (2.15), both the mechanical tension and $t_{A}$ and Maxwell tension $t_{M}^{*}$ are zero; therefore, the boundary conditions are:

$$
\begin{gathered}
\left(\boldsymbol{B}_{3333}+\sigma_{3}\right) u_{r}+p^{*}+\boldsymbol{A}_{33 \mid 3} D_{L_{3}}=0, r=a, b, \\
r v_{r}+u_{\theta}-v=0, r=a, b,(3.20) \\
\boldsymbol{A}_{33 \mid 3}\left(u_{\theta}-v\right)+2 r \boldsymbol{C}_{11} D_{L_{1}}=0 . r=a, b,
\end{gathered}
$$

Note that, by defining $\phi(\theta, r)$ the incompressibility condition (3.5) holds as follows:

$u=\frac{\varphi_{\theta}}{r}, v=-\varphi_{r}$

Similarly, by defining $\psi(\theta, r),(3.8)$ holds for the following ratios:

$D_{L_{3}}=\frac{\psi_{\theta}}{r}, D_{L_{1}}=-\psi_{r}$

However, both $\varphi$ and $\psi$ combine variables. Therefore, the two functions are defined in the following forms to allow for a numerical solution of the principal and boundary equations.

$$
\varphi=r f(r) \sin n \theta, \psi=g(r) \sin n \theta
$$

where $r$ and $\theta$ are defined separately. Therefore, substituting (3.22 -3.24) in (3.16-3.18) produces the below system of differential equations:

$r^{4} \boldsymbol{B}_{3131} f^{\prime \prime \prime \prime}(r)+\left(6 r^{3} \boldsymbol{B}_{3131}+2 r^{4} \boldsymbol{B}_{3131}^{\prime}\right) f^{\prime \prime \prime}(r)+\left(r^{4} \boldsymbol{B}^{\prime \prime}{ }_{3131}+n^{2} r^{2} \boldsymbol{B}_{1111}+5 r^{2} \boldsymbol{B}_{3131}-n^{2} r^{2} \boldsymbol{B}_{3333}-\right.$ $\left.7 r^{3} \boldsymbol{B}_{3131}^{\prime}\right) f^{\prime \prime}(r)+\left(r^{3} \boldsymbol{B}^{\prime \prime}{ }_{3131}-n^{2} r \boldsymbol{B}_{1111}-r \boldsymbol{B}_{3131}-n^{2} r \boldsymbol{B}_{3333}-n^{2} r^{2} \boldsymbol{B}_{1111}^{\prime}+r^{2} \boldsymbol{B}_{3131}^{\prime}-\right.$ $\left.n^{2} r^{2} \boldsymbol{B}_{3333}^{\prime}\right) f^{\prime}(r)+\left(n^{2}-1\right)\left(n^{2} \boldsymbol{B}_{1111}-\boldsymbol{B}_{3131}+r \boldsymbol{B}_{3131}^{\prime}+r^{2} \boldsymbol{B}^{\prime \prime}{ }_{3131}\right) f(r)+\frac{1}{2} r^{2} \boldsymbol{A}_{33 \mid 3} g^{\prime \prime}(r)+$ $\frac{1}{2}\left(r^{2} \boldsymbol{A}_{33 \mid 3}^{\prime}-n^{2} r \boldsymbol{A}_{33 \mid 3}\right) g^{\prime}(r)-n^{2} r \boldsymbol{A}_{33 \mid 3}^{\prime} g(r)=0$

$\frac{1}{2} r^{2} \boldsymbol{A}_{33 \mid 3} f^{\prime \prime}(r)+\left(\frac{1}{2} r\left(n^{2}+2\right) \boldsymbol{A}_{33 \mid 3}+\frac{1}{2} r \boldsymbol{A}_{33 \mid 3}^{\prime}\right) f^{\prime}(r)-\frac{1}{2} r\left(n^{2}-1\right) \boldsymbol{A}_{33 \mid 3}^{\prime} f(r)-r^{2} \boldsymbol{C}_{11} g^{\prime \prime}(r)-r\left(\boldsymbol{C}_{11}\right.$ $\left.+r \boldsymbol{C}_{11}^{\prime}\right) g^{\prime}(r)+n^{2} \boldsymbol{C}_{11} g(r)=0(3.26)$ 
Further, the three boundary conditions corresponding to the above equations are:

$$
\begin{aligned}
r^{3} \boldsymbol{B}_{3131} f^{\prime \prime \prime}(r) & +\left(4 r^{2} \boldsymbol{B}_{3131}+r^{3} \boldsymbol{B}_{3131}^{\prime}\right) f^{\prime \prime}(r) \\
& +\left(r^{2} \boldsymbol{B}_{3131}^{\prime}-n^{2} r^{2} \boldsymbol{B}_{1111}+r \boldsymbol{B}_{3131}-n^{2} r \boldsymbol{B}_{3131}-n^{2} r \boldsymbol{B}_{3333}\right) f^{\prime}(r) \\
& +\left(n^{2}-1\right)\left(\boldsymbol{B}_{3131}+r \boldsymbol{B}_{3131}^{\prime}\right) f(r)-n^{2} \boldsymbol{A}_{33 \mid 3} g(r)+\frac{1}{2} r \boldsymbol{A}_{33 \mid 3}^{\prime} g^{\prime}(r)=0
\end{aligned}
$$

$2 r \boldsymbol{C}_{11} g^{\prime}(r)-2 r \boldsymbol{A}_{33 \mid 3} f^{\prime}(r)+\left(n^{2}-1\right) f(r)=0$

$r^{2} f^{\prime \prime}(r)+r f^{\prime}(r)+\left(n^{2}-1\right) f(r)=0$

Note that, the following equations were used to obtain $(3.25-3.29)$.

$p^{\prime}=\boldsymbol{B}_{3131}^{\prime}-\boldsymbol{B}_{1331}^{\prime}+\frac{\boldsymbol{B}_{3131}-\boldsymbol{B}_{1313}}{r}$,

$p^{\prime \prime}=\boldsymbol{B}^{\prime \prime}{ }_{3131}-\boldsymbol{B}^{\prime \prime}{ }_{1331}+\frac{\boldsymbol{B}_{3131}-\boldsymbol{B}_{1313}}{r^{2}}+\frac{\boldsymbol{B}_{3131}-\boldsymbol{B}_{1313}}{\boldsymbol{r}}$.

\subsection{Problem Non dimensionalization}

The following dimensionless parameters are defined to help achieve the objectives and numerical results.

$$
\begin{gathered}
\hat{r}=\frac{r}{A}, \hat{f}(\hat{r})=\frac{f(r)}{A}, \hat{g}(\hat{r})=\frac{g(r)}{\sigma_{f a} A}, \hat{\sigma}_{f a}{ }^{2}=\frac{\sigma_{f a}{ }^{2}}{\mu \varepsilon} \\
\widehat{\boldsymbol{B}}=\frac{\boldsymbol{B}}{\mu}, \widehat{\boldsymbol{A}}=\boldsymbol{A} \frac{\varepsilon}{\sigma_{f a}}, \widehat{\boldsymbol{C}}=\frac{\boldsymbol{C}}{A}(3.30)
\end{gathered}
$$

Further, given the nature of the problem, it is found that the deformation gradient tensor $F$ is as follows.

$$
[F]=\left[\begin{array}{ccc}
r / R & 0 & 0 \\
0 & 1 & 0 \\
0 & 0 & d r / d R
\end{array}\right]
$$

The principal stretches are, therefore, as follows:

$\lambda_{1}=\frac{r}{R}=\frac{r}{\sqrt{k+r^{2}}}, \lambda_{2}=1, \lambda_{3}=\frac{R}{r}=\frac{\sqrt{k+r^{2}}}{r}$,

In the numerical calculations, a simple strain energy function can be used for an electro-elastic material corresponding to the neo-Hookean, thus:

$W\left(I_{1}, I_{5}\right)=\frac{\mu}{2}\left(I_{1}-3\right)+\frac{1}{2 \varepsilon} I_{5}$

where $\mu=\varepsilon=1$.

Further, electro-elastic moduli are obtained as follows:

$$
\begin{aligned}
\boldsymbol{B}_{3131}=\boldsymbol{B}_{3333}=\lambda_{3}{ }^{2}+D_{r}(a)^{2} \sigma_{f a}{ }^{2} & =\frac{r^{2}+k}{r^{2}} D_{r}(a)^{2} \sigma_{f a}{ }^{2}, \\
\boldsymbol{B}_{1313} & =\boldsymbol{B}_{1111}=\lambda_{1}{ }^{2}=\frac{r^{2}}{r^{2}+k}, 2 \boldsymbol{A}_{13 \mid 1}=\boldsymbol{A}_{33 \mid 3}=2 D_{r}(a),
\end{aligned}
$$


$\boldsymbol{C}_{11}=\boldsymbol{C}_{33}=1, D_{r}(a)=\frac{a}{r}$

Finally, the following system of (non dimensionalized) equations is obtained using Mathematica software:

$$
\begin{aligned}
\left(\frac{3\left(n^{2}-1\right)}{r^{4}}+\frac{4\left(r^{2}-k n^{2}+k\right)+n^{2} r^{2}\left(n^{2}-5\right)}{r^{2}\left(k+r^{2}+a^{2} \sigma_{f a}^{2}\right)}\right) f(r) & \\
& +\left(\frac{n^{2}+3}{r^{3}}+\frac{2 k\left(4 r^{2}+k n^{2}+2 k\right)+r^{2}\left(3 n^{2} r^{2}+4 r^{2}-7 k n^{2}\right)}{r\left(k+r^{2}\right)^{2}\left(k+r^{2}+a^{2} \sigma_{f a}^{2}\right)}\right) f^{\prime}(r) \\
& +\left(\frac{8\left(k+r^{2}\right)-n^{2} r^{2}}{\left(k+r^{2}\right)\left(k+r^{2}+a^{2} \sigma_{f a}{ }^{2}\right)}-\frac{n^{2}+3}{r^{2}}\right) f^{\prime \prime}(r)+\left(\frac{1}{a r}-\frac{k+r^{2}}{a r\left(k+r^{2}+a^{2} \sigma_{f a}{ }^{2}\right)}\right) g^{\prime \prime}(r) \\
& +\left(\frac{\left(n^{2}+1\right)\left(k+r^{2}\right)}{a r^{2}\left(k+r^{2}+a^{2} \sigma_{f a^{2}}{ }^{2}\right.}-\frac{n^{2}+1}{a r^{2}}\right) g^{\prime}(r)+\left(\frac{2 n^{2}}{a r^{3}}-\frac{2 n^{2}\left(k+r^{2}\right)}{a r^{3}\left(k+r^{2}+a^{2} \sigma_{f a}{ }^{2}\right)}\right) g(r) \\
& +\left(\frac{2}{r}-\frac{4 r}{k+r^{2}+a^{2} \sigma_{f a}^{2}}\right) f^{\prime \prime \prime}(r)+f^{\prime \prime \prime \prime}(r)=0,(3.35) \\
\frac{a\left(1-n^{2}\right)}{r^{3}} & f(r)-\frac{a\left(1+n^{2}\right)}{r^{2}} f^{\prime}(r)-\frac{a}{r} f^{\prime \prime}(r)-\frac{n^{2}}{r^{2}} g(r)+\frac{1}{r} g^{\prime}(r)+g^{\prime \prime}(r)=0,(3.36)
\end{aligned}
$$

Similarly, boundary conditions (non dimensionalized) are as follows:

$$
\begin{gathered}
\left(\frac{1-n^{2}}{r^{3}}+\frac{2\left(n^{2}-1\right)}{r\left(k+r^{2}+a^{2} \sigma_{f a^{2}}\right)}\right) f(r)+\left(\frac{2 n^{2}\left(k+r^{2}\right)}{a r^{2}\left(k+r^{2}+a^{2} \sigma_{f a^{2}}\right)}-\frac{2 n^{2}}{a r^{2}}\right) g(r)+\left(\frac{2\left(k+r^{2}\right)-n^{2} r^{2}}{\left(k+r^{2}\right)\left(k+r^{2}+a^{2} \sigma_{f a}{ }^{2}\right)}-\frac{2 n^{2}+1}{r^{2}}\right) f^{\prime}(r)+\left(\frac{1}{a r}-\right. \\
\left.\frac{k+r^{2}}{a r\left(k+r^{2}+a^{2} \sigma_{f a^{2}}\right)}\right) g^{\prime}(r)+\left(\frac{2}{r}-\frac{2 r}{k+r^{2}+a^{2} \sigma_{f a^{2}}}\right) f^{\prime \prime}(r)+f^{\prime \prime \prime}(r)=0(3.37) \\
f^{\prime \prime}(r)+\frac{1}{r} f^{\prime}(r)+\frac{n^{2}-1}{r^{2}} f(r)=0,(3.38) \\
g^{\prime}(r)-\frac{a}{r} f^{\prime}(r)+\frac{a\left(n^{2}-1\right)}{r^{2}} f(r)=0,(3.39)
\end{gathered}
$$

\section{Numerical Results}

This section is divided to three parts. part 1 involves briefly compound matrix method, and we use this method for solving governing equations of our problems. part 2 show changes process thickness of cylinder respect to changes of modes. Also, we show changes process thickness with changes of free-surface charge density; i.e. $\sigma_{f a}$.

\section{Compound Matrix Method}

$\mathrm{Ng}$ and Reid (1979) introduced the compound matrix method (CMM) as a solution to Eigen-value and boundary value problems, including ODEs and unstable systems of ODEs. This method deals with a homogeneous system of $2 n$ first-order differential equations written in the following vector form [24]:

$\frac{d y}{d x}=A(x, \lambda) y$,

where $y$ is a $2 n \times 1$ vector and $A$ is a $2 n \times 2 n$ order matrix, which is a function of the independent variable $x$ and $\lambda$. Homogeneous boundary conditions at the beginning and end of the interval are as follows:

$B(x, \lambda) y=0, x=a$

$C(x, \lambda) y=0, x=b(4.3)$ 
where $B$ and $C$ are $2 n \times n$ matrices of the $n$-th order. The solutions are represented with $y^{(i)}$ for $i=1,2,3, \ldots, n$. The general form of the differential equation's solution of (4.1) is:

$$
y=\sum_{i=1}^{n} k_{i} y^{(i)}
$$

where $k_{i}$ are constants. The above is a solution for the Eigen-value problem, provided that a non-zero set $K=\left\{k_{1}, k_{2}, \ldots\right.$, $k_{n}$ \} can be found that satisfies the boundary condition of (4.2-4.3). In the following, the $2 n \times n$ order matrix $M$ is defined where the $i$-th column is made up of $y^{(i)}$, then $y=M K$. Based on the structure of $y^{(i)}$, we have:

$\frac{d M}{d x}=A M$

For matrix $M$, the boundary condition at $x=b$ is as follows:

$$
C(b, \lambda)=\sum_{i=1}^{n} k_{i} y^{(i)}(b)=C M K=0,(4.6)
$$

Given that a non-trivial solution is sought here, the following condition must hold:

$$
\operatorname{det}(C M)=0,(4.7)
$$

however, the direct calculation of the determinant is avoided in the compound matrix method. Instead, the Laplace expansion of the determinant of (4.7) is used:

$$
\operatorname{det}(C M)=\sum_{i=1}^{c(2 n, n)} \psi_{i} \phi_{i}
$$

where $\phi_{i}$ and $\psi_{i}$ are $n \times n$ cofactors of matrices $C$ and $M$ and $c(2 n, n)$ denotes $2 n$ choose $n$.

Therefore, governing equations are rearranged as a system of six ODEs with new variables. For this purpose, new variables are introduced as follows:

$$
\begin{gathered}
y_{1}(r)=f(r), y_{2}(r)=f^{\prime}(r), y_{3}(r)=f^{\prime \prime}(r), \\
y_{4}(r)=f^{\prime \prime \prime}(r), y_{5}(r)=g(r), y_{6}(r)=g^{\prime}(r) .(4
\end{gathered}
$$

Therefore, it construct compound matrix with respect to (3.36) to (3.38) equations. So we have

$$
\begin{gathered}
f^{\prime \prime \prime \prime}(r)+P_{1}(r) f^{\prime \prime \prime}(r)+P_{2}(r) f^{\prime \prime}(r)+P_{3}(r) f^{\prime}(r)+P_{4}(r) f(r)+P_{5}(r) g^{\prime \prime}(r)+P_{6}(r) g^{\prime}(r)+P_{7}(r) g(r)=0 \\
g^{\prime \prime}(r)+Q_{1}(r) g^{\prime}(r)+Q_{2}(r) g(r)+Q_{3}(r) f(r)+Q_{4}(r) f^{\prime}(r)+Q_{5}(r) f^{\prime \prime}(r)=0 .(4.11)
\end{gathered}
$$

Participant with the following boundary conditions:

$$
\begin{gathered}
f^{\prime \prime \prime}(r)+R_{1}(r) f^{\prime \prime}(r)+R_{2}(r) f^{\prime}(r)+R_{3}(r) f(r)+R_{4}(r) g^{\prime}(r)+R_{5}(r) g(r)=0 \\
f^{\prime \prime}(r)+T_{1}(r) f^{\prime}(r)+T_{2}(r) f(r)=0,(4.13) \\
g^{\prime}(r)+N_{1}(r) f^{\prime}(r)+N_{2}(r) f(r)=0,(4.14)
\end{gathered}
$$


where $P_{i}$ 's are coefficients of first main equation and it is:

$$
\begin{gathered}
P_{1}(r)=\frac{2}{r}+\frac{4 r}{k+r^{2}+a^{2} \sigma_{f a}{ }^{2}}, \\
P_{2}(r)=\frac{8\left(k+r^{2}\right)-n^{2} r^{2}}{\left(k+r^{2}\right)\left(k+r^{2}+a^{2} \sigma_{f a}{ }^{2}\right)}-\frac{n^{2}+3}{r^{2}}, \\
P_{3}(r)=\frac{n^{2}+3}{r^{3}}+\frac{2 k\left(4 r^{2}+k n^{2}+2 k\right)+r^{2}\left(3 n^{2} r^{2}+4 r^{2}-7 k n^{2}\right)}{r\left(k+r^{2}\right)^{2}\left(k+r^{2}+a^{2} \sigma_{f a}{ }^{2}\right)}, \\
P_{4}(r)=\frac{3\left(n^{2}-1\right)}{r^{4}}+\frac{4\left(r^{2}-k n^{2}+k\right)+n^{2} r^{2}\left(n^{2}-5\right)}{r^{2}\left(k+r^{2}+a^{2} \sigma_{f a}{ }^{2}\right)}, \\
P_{5}(r)=\frac{1}{a r}-\frac{k+r^{2}}{a r\left(k+r^{2}+a^{2} \sigma_{f a}^{2}\right)^{\prime}} \\
P_{6}(r)=\frac{\left(n^{2}+1\right)\left(k+r^{2}\right)}{a r^{2}\left(k+r^{2}+a^{2} \sigma_{f a}^{2}\right)}-\frac{n^{2}+1}{a r^{2}}, \\
P_{7}(r)=\frac{2 n^{2}}{a r^{3}}-\frac{2 n^{2}\left(k+r^{2}\right)}{a r^{3}\left(k+r^{2}+a^{2} \sigma_{f a}{ }^{2}\right)} \cdot(4.15)
\end{gathered}
$$

$Q_{i}$ 's are coefficients of second main equation and it is:

$$
\begin{gathered}
Q_{1}(r)=\frac{1}{r} \\
Q_{2}(r)=-\frac{n^{2}}{r^{2}}, \\
Q_{3}(r)=\frac{a\left(1-n^{2}\right)}{r^{3}}, \\
Q_{4}(r)=-\frac{a\left(1+n^{2}\right)}{r^{2}}, \\
Q_{5}(r)=-\frac{a}{r} \cdot(4.16)
\end{gathered}
$$

$R_{i}$ 's are coefficients of first boundary condition equation and it is:

$$
\begin{gathered}
R_{1}(r)=\frac{2}{r}-\frac{2 r}{k+r^{2}+a^{2}{\sigma_{f a}}^{2}}, \\
R_{2}(r)=\frac{2\left(k+r^{2}\right)-n^{2} r^{2}}{\left(k+r^{2}\right)\left(k+r^{2}+a^{2} \sigma_{f a}{ }^{2}\right)}-\frac{2 n^{2}+1}{r^{2}}, \\
R_{3}(r)=\frac{1-n^{2}}{r^{3}}+\frac{2\left(n^{2}-1\right)}{r\left(k+r^{2}+a^{2} \sigma_{f a}^{2}\right)^{\prime}}
\end{gathered}
$$




$$
\begin{gathered}
R_{4}(r)=\frac{1}{a r}-\frac{k+r^{2}}{a r\left(k+r^{2}+a^{2} \sigma_{f a}{ }^{2}\right)^{\prime}} \\
R_{5}(r)=\frac{2 n^{2}\left(k+r^{2}\right)}{a r^{2}\left(k+r^{2}+a^{2} \sigma_{f a}{ }^{2}\right)}-\frac{2 n^{2}}{a r^{2}} .
\end{gathered}
$$

Also, other coefficients for third and second boundary condition equation are:

$$
T_{1}(r)=\frac{1}{r}, T_{2}(r)=\frac{n^{2}-1}{r^{2}}
$$

$N_{1}(r)=-\frac{a}{r}, N_{2}(r)=\frac{a\left(n^{2}-1\right)}{r^{2}}$

Now, we can show (4.4) the reduced system to the following form:

$$
y_{1}^{\prime}(r)=y_{2}(r), y_{2}^{\prime}(r)=y_{3}(r), y_{3}^{\prime}(r)=y_{4}(r),
$$

$y^{\prime}{ }_{4}(r)=-\left(P_{4}(r)\right) y_{1}(r)-\left(P_{3}(r)\right) y_{2}(r)-\left(P_{2}(r)\right) y_{3}(r)-\left(P_{5}(r)\right) y_{6}^{\prime}(r)-\left(P_{6}(r)\right) y_{6}(r)-\left(P_{7}(r)\right) y_{5}(r)$ $-\left(P_{1}(r)\right) y_{4}(r)$

$$
y^{\prime}{ }_{5}(r)=y_{6}(r)
$$

$y_{6}^{\prime}(r)=-\left(Q_{3}(r)\right) y_{1}(r)-\left(Q_{4}(r)\right) y_{2}(r)-\left(Q_{5}(r)\right) y_{3}(r)-\left(Q_{2}(r)\right) y_{5}(r)-\left(Q_{1}(r)\right) y_{6}(r) .(4.19)$

Also, we have this reduced system for boundary conditions to the following form:

$$
\begin{aligned}
R_{3}(r) y_{1}(r)+R_{5}(r) y_{5}(r)+R_{2}(r) y_{2}(r)+R_{4}(r) y_{6}(r)+R_{1}(r) y_{3}(r)+y_{4}(r)=0, & \\
& y_{3}(r)+T_{1}(r) y_{2}(r)+T_{2}(r) y_{1}(r)=0, \\
y_{6}(r)+N_{1}(r) y_{2}(r)+N_{2}(r) y_{1}(r)= & 0 .
\end{aligned}
$$

In order to, we could obtained to matrix forms $\frac{d M}{d r}=\mathrm{AM}$ and $\$ C M=0$.

\subsection{Bifurcation analysis and numerical results}

In this section, we show obtained numerical results as compound matrix method with using Mathematica software.

In Figures 1 to 8 , we conclude bifurcation process of $\mu_{1}$ and $\mu_{2}$ respect to $A$ for mode of $n=2,4,6$, 8. in states (I) $\sigma_{f a}=0.0$ \$, (II) $\sigma_{f a}=0.3$, (III) $\sigma_{f a}=0.6$ and (IV) $\sigma_{f a}=0.9$.

Also, we show bifurcation results for $\mu_{1}$ and $\mu_{2}$ for difference free-surface charge destinies $\sigma_{f a}=0.0,0.3,0.6,0.9$ in constant mode $n=20$ in Figures 9 and 10. 


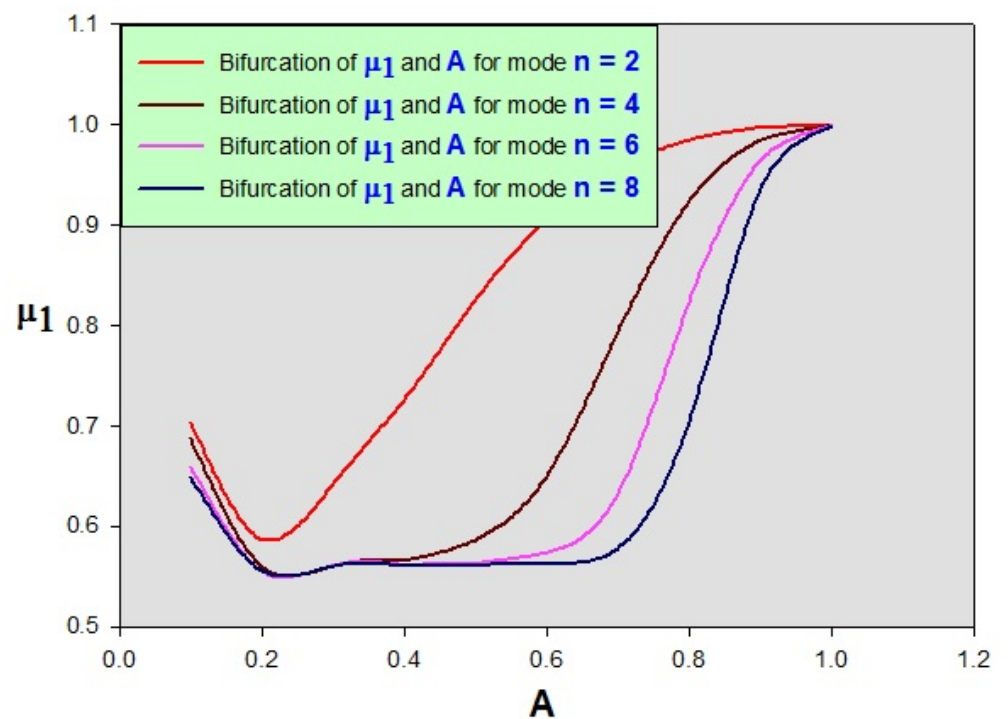

Figure 1. $\mu_{1}$ plotted against $A$ for modes of $n=2,4,6,8$, for charge destiny $\sigma_{f a}=0.0$.

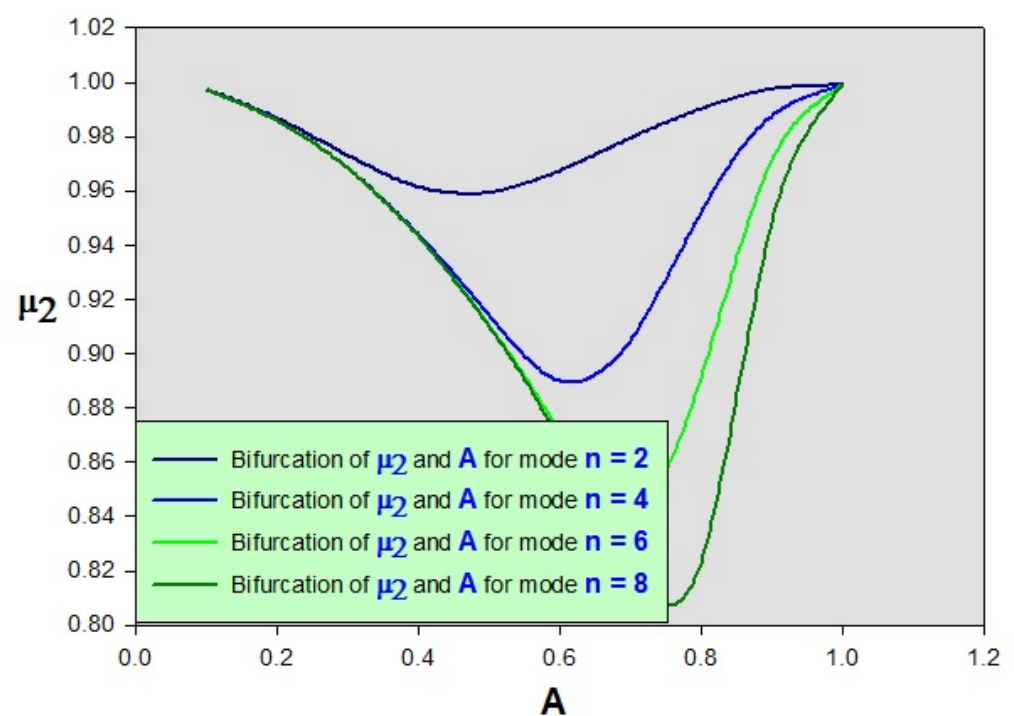

Figure 2. $\mu_{2}$ plotted against $A$ for modes of $n=2,4,6,8$, for charge destiny $\sigma_{f a}=0.0$.

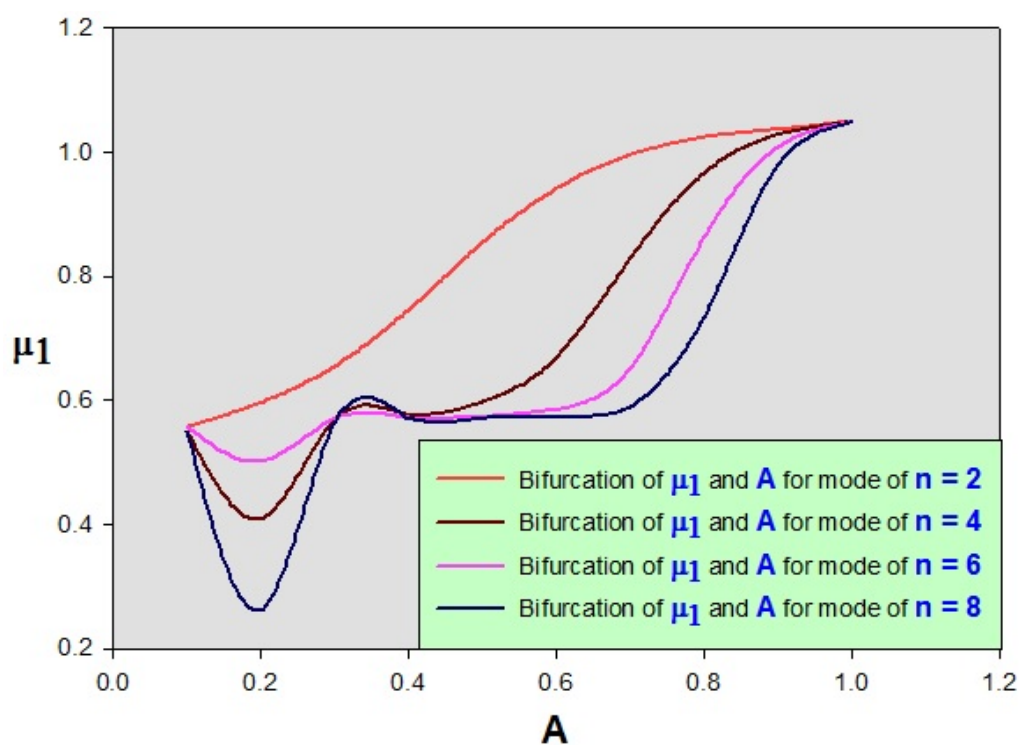

Figure 3. $\mu_{1}$ plotted against $A$ for modes of $n=2,4,6,8$, for charge destiny $\sigma_{f a}=0.3$. 


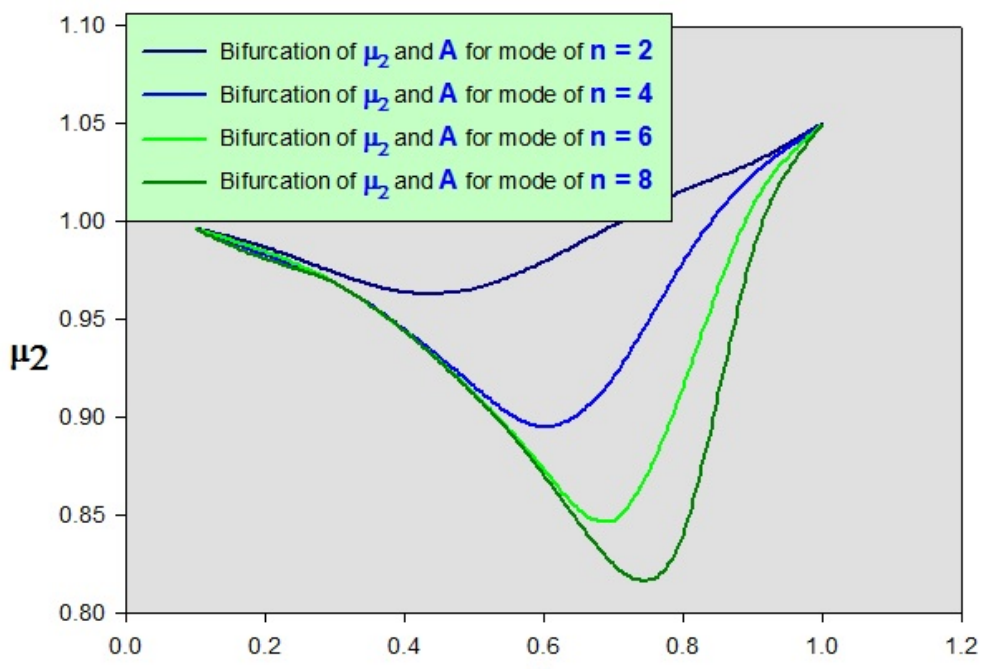

A

Figure 4. $\mu_{2}$ plotted against $A$ for modes of $n=2,4,6,8$, for charge destiny $\sigma_{f a}=0.3$.

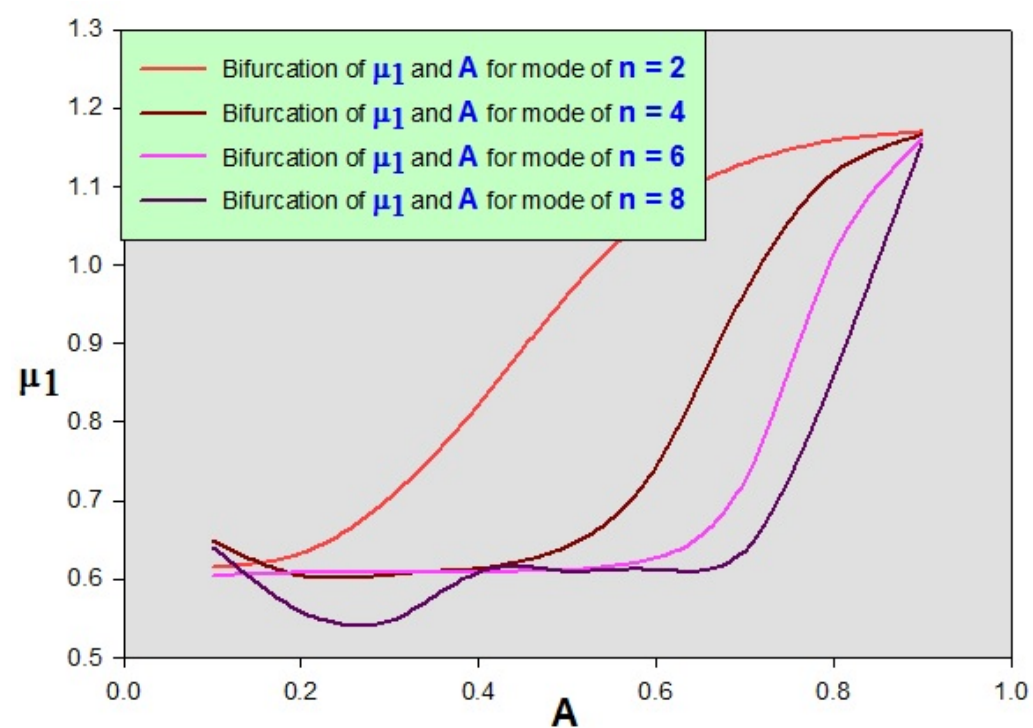

Figure 5. $\mu_{1}$ plotted against $A$ for modes of $n=2,4,6,8$, for charge destiny $\sigma_{f a}=0.6$.

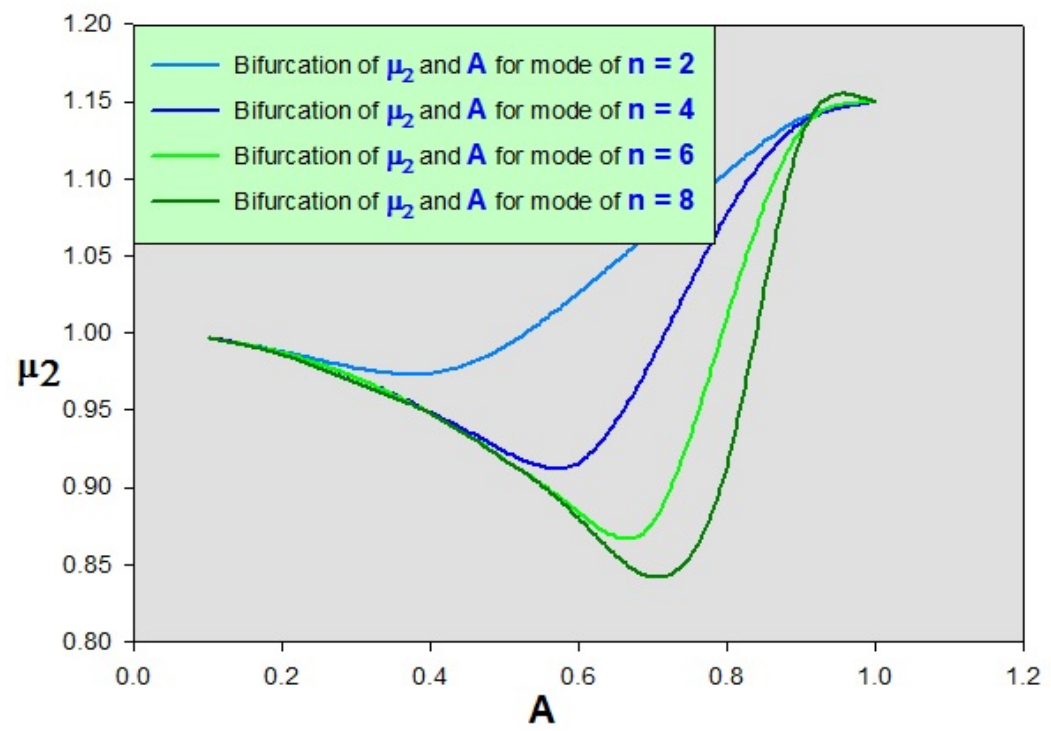

Figure 6. $\mu_{2}$ plotted against $A$ for modes of $n=2,4,6,8$, for charge destiny $\sigma_{f a}=0.6$. 


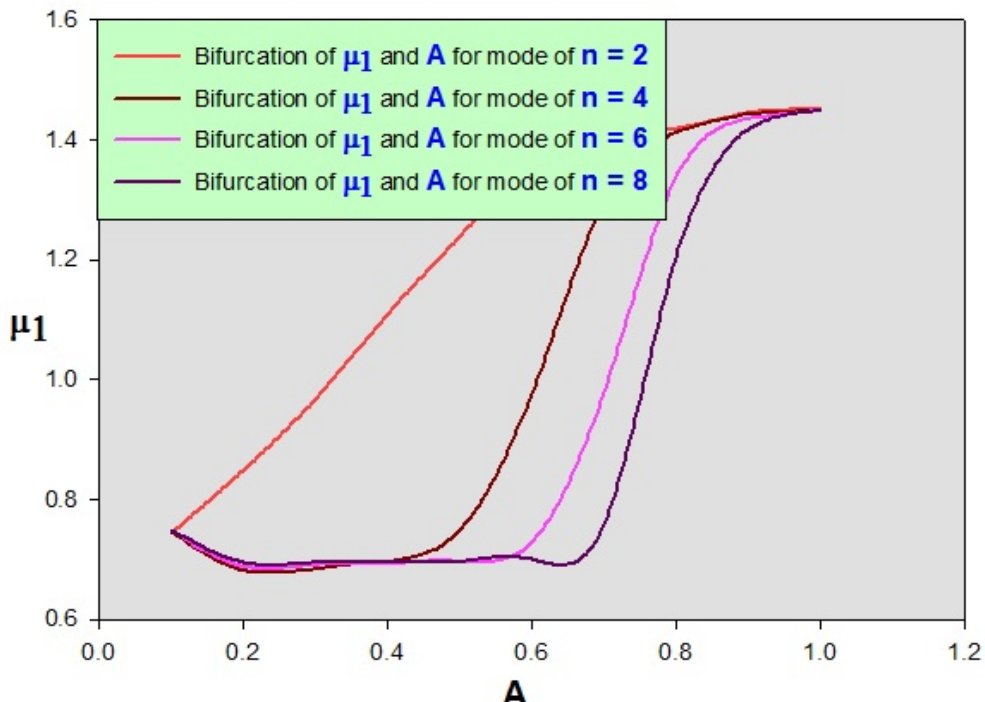

Figure 7. $\mu_{1}$ plotted against $A$ for modes of $n=2,4,6,8$, for charge destiny $\sigma_{f a}=0.9$.

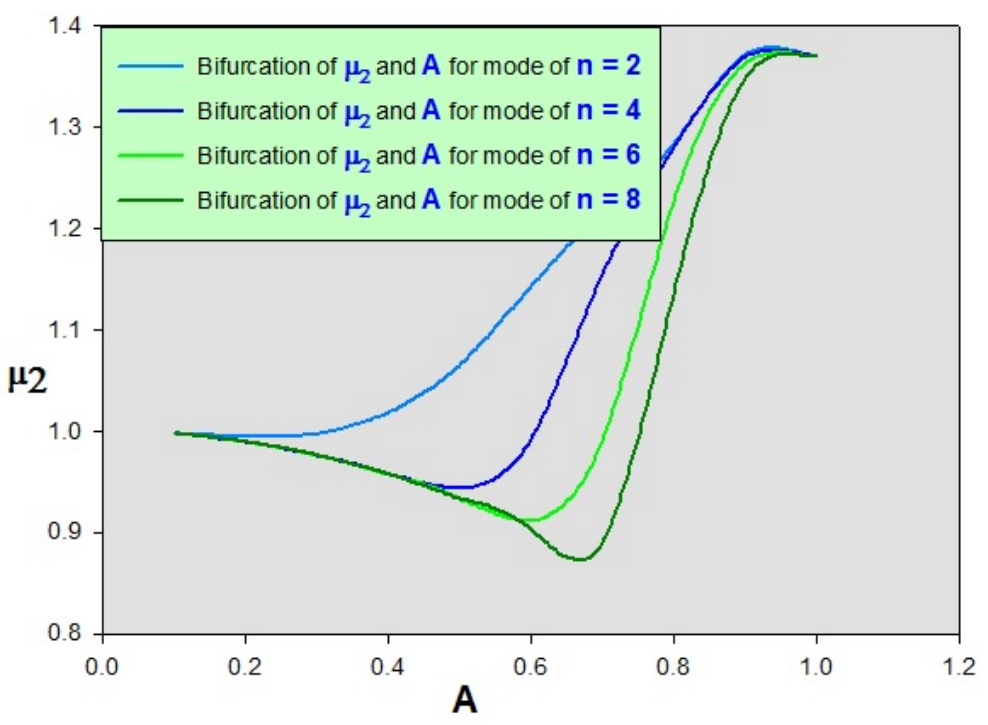

Figure 8. $\mu_{2}$ plotted against $A$ for modes of $n=2,4,6,8$, for charge destiny $\sigma_{f a}=0.9$.

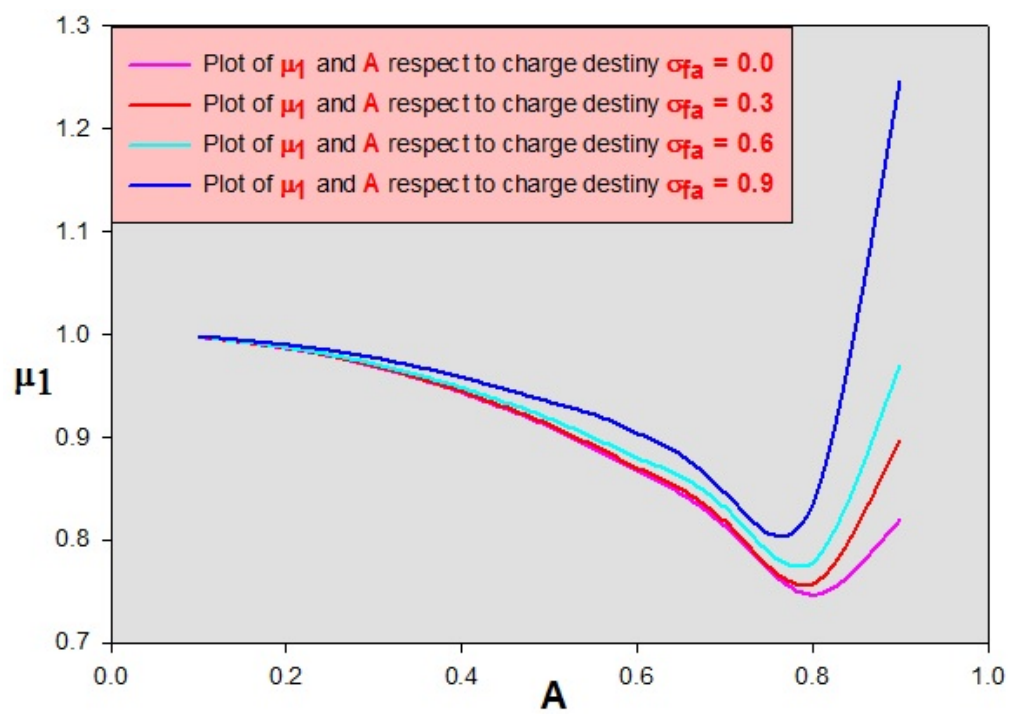

Figure 9. Bifurcation of $\mu_{1}$ respect to $A$ for $\sigma_{f a}=0.0,0.3,0.6,0.9$, for number mode $n=20$. 


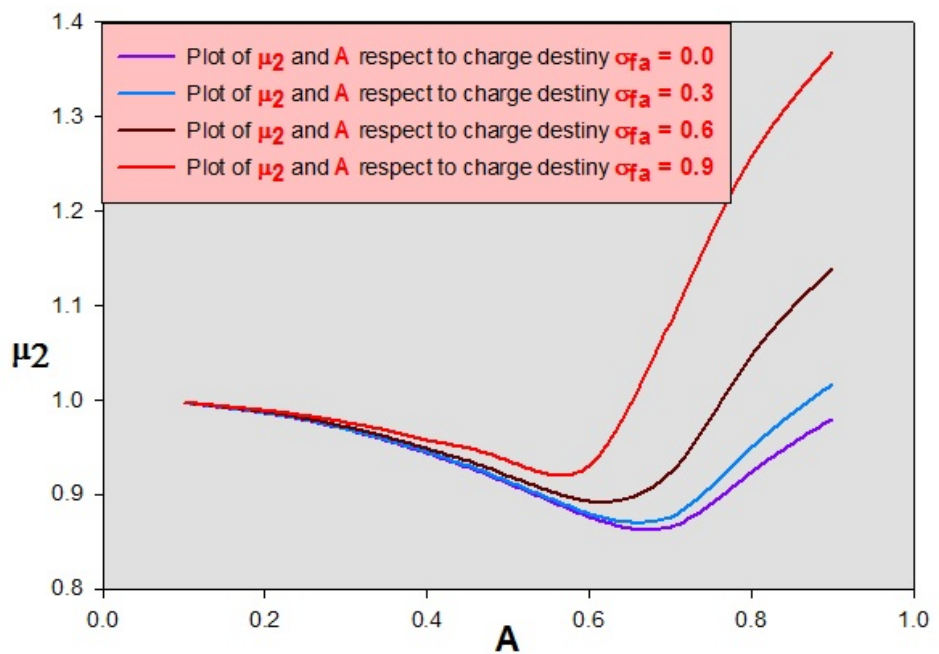

Figure 10. Bifurcation of $\mu_{2}$ respect to $A$ for $\sigma_{f a}=0.0,0.3,0.6,0.9$, for number mode $n=20$.

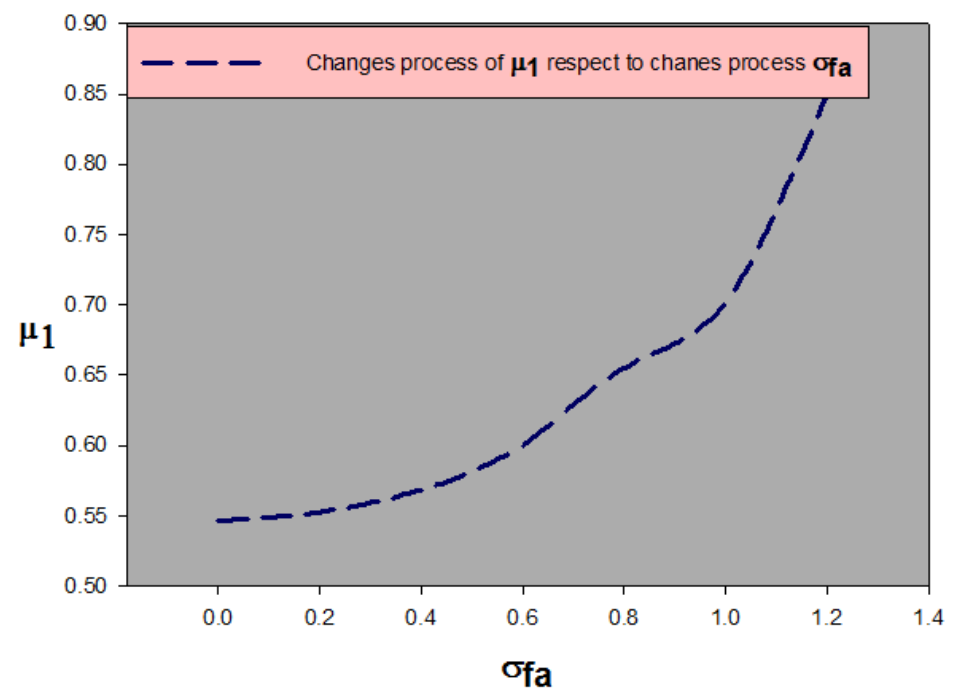

Figure 11. Changes process $\mu_{1}$ with attention to changes of $\sigma_{f a}$

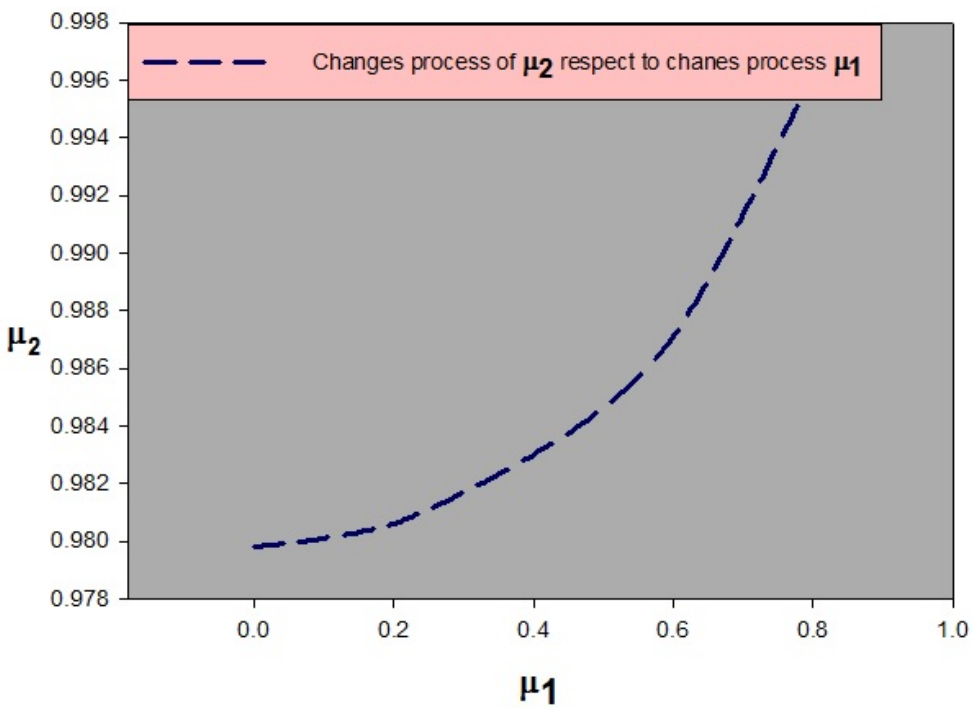

Figure 12. Changes process $\mu_{2}$ with attention to changes of $\mu_{1}$

In Figures 11 and 12, it is showed changes processes $\mu_{1}$ respect to $\sigma_{f a}$ and $\mu_{2}$ respect to $\mu_{1}$. As evident, the bifurcation analysis is carried out based on $A$. The analysis results are presented in Tables 1 and 2 . 
Table 1. Compound matrix solutions of $\mu_{1}$ for different values of $A$ and $n=100$.

\begin{tabular}{|c|c|c|c|c|c|}
\hline \multirow{2}{*}{$A$} & $\mu_{1}$ & $\mu_{1}$ & $\mu_{1}$ & $\mu_{1}$ & $\mu_{1}$ \\
\hline & $\sigma_{f a}=\mathbf{0 . 0}$ & $\sigma_{f a}=0.2$ & $\sigma_{f a}=0.4$ & $\sigma_{f a}=0.6$ & $\sigma_{f a}=0.8$ \\
\hline 0.5 & 0.616005 & 0.599355 & 0.600151 & 0.599919 & 0.957494 \\
\hline 0.53 & 0.500005 & 0.598354 & 0.538688 & 0.599537 & 0.651913 \\
\hline 0.56 & 0.560631 & 0.507016 & 0.598684 & 0.516120 & 0.599669 \\
\hline 0.59 & 0.626707 & 0.600103 & 0.599023 & 0.599421 & 0.650876 \\
\hline 0.62 & 0.600155 & 0.599589 & 0.569425 & 0.601424 & 0.610456 \\
\hline 0.65 & 0.584606 & 0.547435 & 0.649998 & 0.600089 & 0.656912 \\
\hline 0.68 & 0.551917 & 0.500009 & 0.594061 & 0.600000 & 0.550495 \\
\hline 0.71 & 0.507148 & 0.539729 & 0.565126 & 0.599627 & 0.550000 \\
\hline 0.74 & 0.550362 & 0.552027 & 0.568323 & 0.599788 & 0.494906 \\
\hline 0.77 & 0.546872 & 0.552007 & 0.568439 & 0.599866 & 0.655166 \\
\hline 0.80 & 0.546872 & 0.552007 & 0.568439 & 0.599866 & 0.655166 \\
\hline 0.83 & 0.546872 & 0.552007 & 0.568439 & 0.599866 & 0.655166 \\
\hline 0.86 & 0.546872 & 0.552007 & 0.568439 & 0.599866 & 0.655166 \\
\hline 0.89 & 0.546872 & 0.552007 & 0.568439 & 0.599866 & 0.655166 \\
\hline 0.92 & 0.546873 & 0.552008 & 0.568440 & 0.599867 & 0.655167 \\
\hline 0.95 & 0.547275 & 0.552431 & 0.568938 & 0.600015 & 0.655807 \\
\hline
\end{tabular}

Table 2. Compound matrix solutions of $\mu_{2}$ for different values of $A$ and $n=100$.

\begin{tabular}{|c|c|c|c|c|c|}
\hline \multirow{2}{*}{$A$} & $\mu_{1}$ & $\mu_{1}$ & $\mu_{1}$ & $\mu_{1}$ & $\mu_{1}$ \\
\hline & $\sigma_{f a}=\mathbf{0 . 0}$ & $\sigma_{f a}=0.2$ & $\sigma_{f a}=0.4$ & $\sigma_{f a}=0.6$ & $\sigma_{f a}=0.8$ \\
\hline 0.5 & 0.919174 & 0.916410 & 0.916540 & 0.915502 & 0.989545 \\
\hline 0.53 & 0.888440 & 0.905356 & 0.916540 & 0.905576 & 0.915685 \\
\hline 0.56 & 0.885983 & 0.875794 & 0.894770 & 0.905576 & 0.893964 \\
\hline 0.59 & 0.888043 & 0.881623 & 0.893757 & 0.877460 & 0.894074 \\
\hline 0.62 & 0.868364 & 0.868213 & 0.881367 & 0.881461 & 0.871119 \\
\hline 0.65 & 0.849644 & 0.839117 & 0.869486 & 0.868701 & 0.871678 \\
\hline 0.68 & 0.823682 & 0.808210 & 0.837129 & 0.854193 & 0.823245 \\
\hline 0.71 & 0.790920 & 0.801716 & 0.810486 & 0.839085 & 0.805227 \\
\hline 0.74 & 0.786300 & 0.786939 & 0.793265 & 0.822892 & 0.765849 \\
\hline 0.77 & 0.764472 & 0.766657 & 0.773744 & 0.805852 & 0.816687 \\
\hline 0.80 & 0.742566 & 0.744994 & 0.752860 & 0.768308 & 0.796690 \\
\hline 0.83 & 0.719117 & 0.721814 & 0.730547 & 0.747659 & 0.778977 \\
\hline 0.86 & 0.693968 & 0.696968 & 0.706669 & 0.725629 & 0.760176 \\
\hline 0.89 & 0.666928 & 0.670270 & 0.681062 & 0.702089 & 0.740205 \\
\hline 0.92 & 0.637756 & 0.641490 & 0.653523 & 0.676882 & 0.718966 \\
\hline 0.95 & 0.606472 & 0.610676 & 0.624204 & 0.649936 & 0.696886 \\
\hline
\end{tabular}

\section{Conclusion}

The present study investigates the bending of a simple electro-elastic cylindrical shell of neo-Hookean material placed under external hydrostatic pressure and exposed to an electric field. Further, after modeling the problem, the compound matrix method was used to analyze the bifurcation of $\mu_{1}$ and $\mu_{2}$ using the Eigen-value problem. According to Table 1, for a numerical mode number of 100 when $\sigma_{f a}$ is zero (that is, electric displacement has the least impact on the electric field), $\mu_{1}$ values fluctuate from $A=0.5$ to 0.75 . However, from $A=0.75$ to $0.95, \mu_{1}$ does not exceed $\sigma_{f a}$. For $\sigma_{f a}=$ 0.2 , similarly, $\mu_{1}$ fluctuates in the $A=0.5$ to $A=0.75$ range, but from $A=0.75$ to $0.95, \mu_{1}$ converges to 0.5520 . The same goes for $\sigma_{f a}=0.4,0.6$, and 0.8 , as $\mu_{1}$ approaches $0.5684,0.5998$, and 0.6551 . However, no conclusion can be made regarding $\mu_{2}$ variations by increasing $\sigma_{f a}$ from 0 to 0.8 and for $n=100$; since, as evident from Table 2, $\mu_{2}$ changes according to $A$ and $\mu_{1}$, whereas $\mu_{1}$ depends only on $A$ and $\sigma_{f a}$. 


\section{Acknowledgments}

The authors would like to extend gratitude to Andrey Melnikov for their invaluable contributions to the theory of nonlinear electro-elasticity, pushing the boundaries in mathematics and physics.

Author contributions: Conceptualization, O Teymoori and A Hatami; Methodology, O Teymoori and A Hatami; Investigation, O Teymoori and A Hatami; Writing - original draft, O Teymoori; Writing - review \& editing, A Hatami; Funding acquisition, A Hatami; Resources, O Teymoori; Supervision, A Hatami.

\section{Editor: Rogério José Marczak}

\section{References}

Tiersten, H., F. (1971), “On the Nonlinear Equations of Thermoelectroelasticity“, Int. J. Engng. Sci., 9, 587.

Dorfmann, A., Ogden, R., W. (2005), “Nonlinear electro-elasticity", Acta Mechanica, 174, 167-183.

Fiebig, M. (2005), “Revival of the magneto-electric effect “, J. Phys. D-Appl. Phys., 38, (8), 123-152.

Toupin, R., A.,(1956). “The elastic dielectric “, Journal of Rational Mechanics Analysis, 5, 849-914.

Zharii, O., Y.(1990), "The Eigen-function expansion method in dynamic electro-elasticity problems", PMM U. S. S. R, Volume 54 , No $1,88-93$.

Dieulesiant, E., Royer, D. (1981), "Elastic waves in solids": Applications to Signal Processing Wiley-Inter Science. New York.

Shul'ga, V., M. (2005), "Nonaxisymmetric electro-elastic vibrations of a hollow cylinder with radial axes of physic-mechanical symmetry", International Applied Mechanics, 41, No 7, 766-769.

Eva, L., O., G. (2006), "Numerical solution to the initial-boundary-value problem of electro-elasticity for a radially polarized hollow piezo-ceramic cylinder", International Applied Mechanics, Volume 42, No 12, 67-75.

Eva, L., O., G., Shul'ga, N., A. (2008), "Solution of initial-boundary-value problems of electro-elasticity revisited ", International Applied Mechanics, Volume 44, No 12, 62-69.

Melnikov, A., Ogden, R., W. (2016), “Finite deformations of an electro-elastic circular cylindrical tube”, Z. Angew. Math. Phys., 67-140.

Bar-Cohen, Y.(2001), “Electro-active polymer (EAP) actuators as Artificial Muscles”, SPIE Press. Bellingham. WA., 3-44.

Mckay, T., O'Brien, B., Calius, E., Anderson, I.(2010), “An integrated self-priming dielectric elastomer generator”, Applied Physics and Letters, 97.

Rudykh, S., Bhattacharya, K., deBotton, G.(2012), "Snap-through actuation of thick-wall electro-active balloons", International Journal of Nonlinear Mechanics, 47, 206-209.

Shumel, G., Gei, M., deBotton, G.(2012), "The Rayleigh-Lamb wave propagation in dielectric elastomer layers subjected to large deformations", International Journal Nonlinear Mechanics, 47, 307-316.

Dorfmann, A., Ogden, R., W. (2010), “Electro-elastic waves in a finitely deformed electro-active material”, IMA. Journal Applied Mathematics, 75, 603-636.

Wang, Y., Li, Z., Chen, W., Q., Zhang, C., L. (2017), “On guided circumferential waves in soft electro-active tubes under radially inhomogeneous biasing fields", Journal of Mechanics and Physics Solids, 99, 116-145.

Dorfmann, L., Ogden, R., W. (2017), "Nonlinear electro-elasticity: Material properties, continuum theory and applications", The Royal Society Publishing, 1-34.

Melnikov, A.(2016), "Bifurcation of thick-walled electro-elastic cylindrical and spherical shells at finite deformation", PhD. Thesis, University of Glasgow, 2016.

Haughton, D., M., Ogden, R., W. (1978), “On the incremental equations in nonlinear elasticity-II: Bifurcation of pressurized spherical shells", Journal of Mechanics and Physics Solids, (26), 111-138. 
Liu, L. (2013), "An energy formulation of continuum magneto-electro-elasticity with applications”, Journal of Mechanics and Physics Solids, 63, 451-480.

Melnikov, A., Dorfmann, L., Ogden, R., W. (2020), "Bifurcation of finitely deformed thick-walled electro-elastic spherical shells subject to a radial electric field", International Journal of nonlinear Mechanics, 121.

Su,Y., Wu, B., Chen, W., Destrade, M.(2019), "Pattern evolution in bending dielectric- elastomeric bilayers", Journal of the Mechanics and Physics of Solids, 136.

Broderick, H., C., Dorfmann, L., Destrade, M. (2020), “Electro-elastic Lamb waves in dielectric plates”, Extreme Mechanics Letters, 1-14.

Ng, B., S., Reid, W., H. (1979), " A numerical method for linear two-point boundary-value problems using compound matrices", Journal of Computational Physics, 33, 70-85.

Melnikov, A., Ogden, R.,W. (2018), "Bifurcation of finitely deformed thick-walled electro-elastic cylindrical tubes subject to a radial electric field", Z. Angew. Math. Phys., 60-69.

Cohen, N., Dayal, K., deBotton, G. (2016), “Electro-elasticity of polymer networks”, Journal of the Mechanics and Physics of Solids, 92, 105-126.

Sanjaranipour, M., Hatami, A., Abdolalian, N. (2013), "Another approach of WKB method for the stability analysis of the bending of an elastic rubber block", International Journal of Engineering Science, Vol. 62, 1-8. 